\title{
AVALIAÇÃO DE FOCO EPILEPTOGÊNICO DO LOBO TEMPORAL: CORRELAC̣ÃO ENTRE SPECT ICTAL, RESSONÂNCIA MAGNÉTICA E RESSONÂNCIA MAGNÉTICA COM ESPECTROSCOPIA DE PRÓTONS*
}

\author{
Maria Elena Martins Diegues ${ }^{1}$, Marcos Pinto Pellini ${ }^{2}$, Soniza Vieira Alves-Leon ${ }^{3}$, Romeu Côrtes \\ Domingues $^{4}$
}

Resumo 0 objetivo deste trabalho foi determinar a existência de concordância entre os métodos radioisotópico e radiológico e, em caso positivo, avaliar a utilidade do SPECT ictal na determinação do foco epileptogênico. Foram realizados SPECT ictal, ressonância magnética (RM) e ressonância magnética com espectroscopia de prótons (RME) em seis pacientes com epilepsia de lobo temporal refratária. O SPECT ictal foi realizado após a retirada das drogas antiepilépticas durante monitoramento por vídeo-EEG, utilizando-se o ${ }^{99 m} T c-E C D$, administrado aos pacientes no início da crise. As imagens de RM foram obtidas em T1, T2 e FLAIR, com cortes de 3 e $5 \mathrm{~mm}$ de espessura, e a RME foi realizada com técnica PRESS, com voxel único posicionado no hipocampo, bilateralmente. A análise estatística incluiu os valores de Kappa (k), erro-padrão (ep) e o nível de significância (p) para a lateralização do foco. Os achados foram analisados com base na localização por EEG da descarga ictal, no tempo de duração da crise (109-280 s; média: 152 s) e no tempo de administração do traçador (30-262 s; média: 96 s). Obtivemos dados correlatos em quatro pacientes (67\%), com valores de $k=0,67$, ep $=0,38$ e $p=0,041$. Concluímos que existe concordância entre SPECT ictal, RM e RME, e a utilidade do procedimento radioisotópico está relacionada aos casos em que o EEG não é diagnóstico e quando há discordância ou indefinição diagnóstica na análise comparativa entre EEG, RM e RME. Unitermos: SPECT ictal; Ressonância magnética; Espectroscopia; Epilepsia.

Abstract Evaluation of epileptogenic focus in temporal lobe: correlation between ictal brain SPECT, magnetic resonance imaging and magnetic resonance spectroscopy.

The purpose of this study was to determine the degree of concordance between radiological and radioisotopic methods and, if positive, to evaluate the usefulness of ictal SPECT in the localization of the epileptogenic focus. Ictal brain SPECT, magnetic resonance imaging (MRI) and magnetic resonance spectroscopy (MRS) were performed on six patients with refractory temporal lobe epilepsy. Ictal SPECT was performed after withdrawal of the anti-epileptogenic drugs during video-EEG monitoring, using ${ }^{99 m} T c-E C D$, administered to patients at the time of the ictus. MRI was performed in T1, T2 and FLAIR sequences and MRS was obtained using the PRESS technique, with a single voxel positioned in both hippocampi. The statistical analysis included the determination of the values of Kappa (k), standard error (se) and significance level (p) for the lateralization of the ictal focus. The analysis of all findings was based on EEG localization of the ictal discharge, seizure duration (109-280 s; $152 \mathrm{~s}$ average) and time of radiotracer injection (30-262 s; $96 \mathrm{~s}$ average). We obtained correlated data in four patients $(67 \%)$, and values of $k=0.67$, se $=0.38$, and $p=$ 0.041 . We concluded that there is a concordance between ictal SPECT, MRI and MRS data and the usefulness of the radioisotopic procedure is related to a nondiagnostic EEG and when there is a discordant or misleading diagnosis after a comparative analysis of EEG and MRS.

Key words: Ictal SPECT; Magnetic resonance imaging; Magnetic resonance spectroscopy; Epilepsy.

* Trabalho realizado no Serviço de Medicina Nuclear do Hospital Universitário Clementino Fraga Filho (HUCFF) da Universidade Federal do Rio de Janeiro (UFRJ), no Programa de Epilepsias do Serviço de Neurologia do HUCFF-UFRJ e no Instituto de Ressonância Magnética do Rio de Janeiro (IRM), Rio de Janeiro, RJ.

1. Médica Doutoranda do Serviço de Medicina Nuclear do HUCFF-UFRJ.

2. Professor Adjunto Doutor em Radiologia pela UFRJ.

3. Professora Adjunta Doutora em Neurologia pela UFRJ.

4. Radiologista, Diretor das Clínicas CDPI e Multimagem.

Endereço para correspondência: Dra. Maria Elena Martins Diegues. Rua Barão de Lucena, 135/104, Botafogo. Rio de Janeiro, RJ, 22260-020. E-mail: emartyns@terra.com.br

Recebido para publicação em 25/11/2002. Aceito, após revisão, em 4/6/2003.

\section{INTRODUÇÃO}

Definir epilepsia é uma tarefa complexa e difícil. Por epilepsia compreende-se uma ampla categoria de sintomas complexos resultantes de alterações das funções cerebrais, que podem decorrer de inúmeras causas.

Epilepsia é um grupo de doenças que têm em comum crises epilépticas que recorrem na ausência de condição tóxico- metabólica ou febril ${ }^{(\mathbf{1})}$. Crises epilépticas são eventos clínicos que refletem disfunção temporária de um conjunto de neurônios de parte do encéfalo (crises focais) ou de área mais extensa envolvendo simultaneamente os dois hemisférios cerebrais

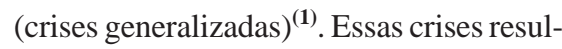
tam de uma descarga excessiva dos neurônios e são caracterizadas por alterações na atividade elétrica, que podem ser registradas pelo eletroencefalograma (EEG). 
Durante uma crise podem ocorrer distúrbios de consciência, dos sistemas sensorial e motor, além de alterações subjetivas do bem-estar e objetivas do comportamento ${ }^{(2)}$. As crises são episódicas, involuntárias, breves, tendo um começo e um fim, também podendo produzir disfunções pós-crise ${ }^{(2)}$.

É a condição de maior prevalência no mundo. Estima-se que $1 \%$ da população mundial apresente essa condição e que o número de novos casos por ano seja da ordem de dois milhões em todo o mundo $^{(\mathbf{3 , 4})}$. Estudos realizados na América Latina, entre 1989 e 1994, estimam uma taxa de prevalência de epilepsia no Brasil da ordem de 13 casos por 1.000 habitantes ${ }^{(\mathbf{5})}$, o que mostra a grande importância médico-social do tema.

A medicina nuclear, com base em avanços recentes nos campos da radiofarmácia e da instrumentação, introduziu na rotina clínica a técnica da tomografia computadorizada por emissão de fóton único (SPECT - "single photon emission computed tomography"), desenvolvida por Kuhl e Edwards ${ }^{(\mathbf{6})}$, em 1963, mediante o uso de uma gama-câmara rotatória.

Desde sua implantação, muitas entidades nosológicas têm sido estudadas esperando-se encontrar dados de utilidade prática. Isto levou o American Academy of Neurology's Therapeutics and Technology Subcommittee a criar um grupo multidisciplinar para revisar a literatura e avaliar a utilidade clínica do SPECT cerebral no diagnóstico e tratamento de enfermidades neurológicas ${ }^{(7)}$. Dentre as aplicações catalogadas como "estabelecidas" encontra-se a epilepsia, sob o aspecto da realização do SPECT ictal para a determinação pré-cirúrgica do foco epileptogênico.

Com base nesses fatos relevantes realizamos, no Hospital Universitário Clementino Fraga Filho (HUCFF) da Universidade Federal do Rio de Janeiro (UFRJ), um estudo objetivando determinar a existência de concordância entre SPECT cerebral ictal, ressonância magnética (RM) e ressonância magnética com espectroscopia de prótons (RME) e, em caso positivo, determinar em que casos o SPECT ictal possui real utilidade para a localização do foco epileptogênico em pacientes com epilepsia de lobo temporal (ELT) refratária ao tratamento clínico.

\section{MATERIAIS E MÉTODOS}

Foram selecionados seis pacientes do sexo masculino, com idades variando entre 34 e 54 anos (média de 41 anos), com ELT refratária, cujo diagnóstico foi feito no Programa de Epilepsias do Serviço de Neurologia do HUCFF, mediante avaliações clínicas, EEG interictal e vídeo-EEG ictal, no período de março a setembro de 2002. Todos os pacientes assinaram o "Termo de consentimento livre e esclarecido" antes de serem incluídos no protocolo do estudo, o qual foi aprovado pelo Comitê de Ética em Pesquisa do HUCFF. Foram excluídos os pacientes que não consentiram na pesquisa ou aqueles em que a avaliação clínica, o EEG interictal e o vídeo-EEG ictal afastaram o diagnóstico clínico de ELT.

Os pacientes selecionados foram submetidos a RM e RME, realizadas por um único radiologista do Instituto de Ressonância Magnética do Rio de Janeiro (IRM), em equipamento GE Signa Horizon de 1,5 T. A RM consistiu nas seqüências T1, T2 e FLAIR, com cortes de $3 \mathrm{~mm}$ de espessura em hipocampo, além de imagens axiais em FLAIR de $5 \mathrm{~mm}$ de espessura para o restante do cérebro. A RME consistiu na técnica PRESS (TR $=1.500 \mathrm{~ms}$ e $\mathrm{TE}=35 \mathrm{~ms}$ ), com voxel único posicionado no hipocampo, bilateralmente.

A interpretação dos dados anatômicos e bioquímicos foi feita por dois radiologistas experientes, sem o conhecimento dos dados clínicos dos pacientes (estudo em paralelo). O diagnóstico de atrofia ou esclerose hipocampal se baseou nos achados de atrofia da formação hipocampal, no aumento da intensidade do sinal da região mesial temporal — demonstrado na imagem em T2 ou na seqüência FLAIR - e na observação de ocasional atrofia amigdalar ou do rinocórtex ${ }^{(\mathbf{8})}$.

Foram avaliadas as relações NAA/Cho $+\mathrm{Cr}$ em ambos os hipocampos e consideradas patológicas quando abaixo de $0,72^{(9)}$.

Para a realização do SPECT ictal os pacientes foram internados no Programa de Epilepsias do Serviço de Neurologia do HUCFF. As drogas antiepilépticas foram retiradas e os pacientes permaneceram monitorizados por vídeo-EEG durante todo o período da internação. A administração endovenosa do radiofármaco, em veia puncionada previamente, foi realizada quando da identificação do início da crise epiléptica através dos traçados do EEG. O tempo decorrido entre o início da crise e a injeção do traçador variou de 30 a 262 segundos (média de 96 segundos), sendo todos injetados no decurso da crise.

O radiofármaco foi preparado no Setor de Radiofarmácia do Serviço de Medicina Nuclear do HUCFF e levado ao Setor de vídeo-EEG, onde permaneceu disponível pelo período de seis horas (tempo de viabilidade do composto radiomarcado). Sua administração aos pacientes ficou a cargo da equipe de enfermagem do Programa de Epilepsias, previamente treinada no Serviço de Medicina Nuclear. A não-utilização do radiofármaco dentro do período de viabilidade implicou o preparo de nova dose.

A todos os pacientes foi administrada a dose de $1.110 \mathrm{MBq}(30 \mathrm{mCi}) \mathrm{de}^{99 \mathrm{~m}} \mathrm{Tc}$ ECD ("ethylcysteinate dimer"), preparado de acordo com as especificações do fabricante, sendo devidamente obedecidas as normas de radioproteção estabelecidas para uso, manuseio e administração de substâncias radioativas em anima nobili.

A interpretação das imagens foi feita por dois médicos nucleares experientes do Serviço de Medicina Nuclear do HUCFF, sem o conhecimento dos resultados dos demais exames. O critério de interpretação foi a identificação de área de hiperfixação do radiofármaco em determinado hemisfério cerebral quando comparada à mesma área no hemisfério contralateral ${ }^{(\mathbf{1 0 , 1 1})}$.

O SPECT ictal foi realizado cerca de 30 a 45 minutos após a administração do radiotraçador, em gama-câmara Anger rotatória de duplo detector GE Millenium MG.

O tempo total de aquisição foi de $21 \mathrm{mi}$ nutos e 40 segundos. Foram adquiridas 64 projeções, de 20 segundos cada, em matriz $64 \times 64$, com rotação circular de $360^{\circ}$ a partir da posição anterior $\left(0^{\circ}\right)$. Utilizou-se colimador LEHR ("low energy high resolution") e as imagens foram adquiridas com fator de aumento ("zoom" = 2.00).

$\mathrm{O}$ processamento das imagens foi feito por correção de "scatter" e retroprojeção com filtros Ramp e Butterworth de ordem 10 e freqüência de corte de 0,40 Ny. Os cortes transversos originais tiveram alinhamento órbito-meatal e correção de atenuação pelo método de Chang, com coefi- 
ciente de atenuação de $0,12 \mathrm{~cm}^{-1}$. Os cortes tomográficos finais, nos planos transversal, sagital e coronal, foram obtidos com espessura de 1 pixel.

Os resultados foram tratados pela estatística Kappa (k). Esta estatística mede o grau de concordância para dados qualitativos, isto é, quanto maior o valor de k, mais forte é a concordância entre as duas técnicas utilizadas. Kappa igual a 1 expressa concordância perfeita. O critério de determinação de significância foi de $5 \%$, ou seja, se o p valor do teste for menor ou igual a 0,05, então existe significância estatística (intervalo de confiança de 95\%).

\section{RESULTADOS}

Com base na localização eletroencefalográfica da descarga neuronal inicial, no tempo de duração da crise, no tempo de administração do radiofármaco e nos resultados da RM, da RME e do SPECT ictal, encontramos $^{(\mathbf{1 2})}$ :

1 - Alterações anatômicas e/ou bioquímicas uni ou bilaterais na RM e RME, levando-se em conta a dominância das alterações bilaterais com resultado concordante do SPECT ictal em quatro casos $(67 \%)$ :

a) Caso 1 - descarga em lobo temporal direito, esclerose mesial hipocampal direita e relação NAA/Cho+Cr normal $(0,74$ à direita e 0,88 à esquerda): o SPECT ictal mostrou hiperfixação do radiofármaco em região fronto-temporal direita.

b) Caso 2 - descarga fronto-temporal à esquerda, esclerose mesial hipocampal bilateral e relação NAA/Cho+Cr reduzida bilateralmente, com predomínio à esquerda $(0,51$ contra 0,56 à direita): o SPECT ictal mostrou hiperfixação do radiofármaco em lobo temporal esquerdo.

c) Caso 3 - descarga temporal bilateral, ausência de esclerose mesial hipocampal e relação NAA/Cho+Cr reduzida bilateralmente, com predomínio à direita $(0,64$ contra 0,66 à esquerda): o SPECT ictal mostrou hiperfixação do radiofármaco em região fronto-temporal direita.

d) Caso 4 - descarga temporal bilateral, com predominância à direita na crise estudada, esclerose mesial hipocampal direita (Figura 1A) e relação NAA/Cho+Cr reduzida bilateralmente, com discreto predomínio à esquerda $(0,47$ contra 0,48 à direita) (Figura 1B): o SPECT ictal mostrou hiperfixação do radiofármaco em lobo temporal direito (Figura 1C).

2 - Alterações anatômicas e/ou bioquímicas uni ou bilaterais na RM e RME, levando-se em conta a dominância das alterações bilaterais, com resultado discordante do SPECT ictal em dois casos (33\%):

a) Caso 5 - descarga temporal bilateral, ausência de esclerose mesial hipocampal (Figura 2A) e relação NAA/Cho+Cr reduzida em hipocampo direito $(0,68$ contra 0,90 à esquerda) (Figura 2B): o SPECT ictal mostrou hiperfixação do radiofármaco em lobo temporal esquerdo (Figura 2C).

b) Caso 6-descarga em lobo temporal direito, esclerose mesial hipocampal direita e relação NAA/Cho+Cr reduzida bilateralmente, com predomínio à direita $(0,61$ contra 0,69 à esquerda): o SPECT ictal mostrou hiperfixação do radiofármaco em lobo temporal esquerdo.

Para esses resultados foram calculados os valores de k, erro padrão (ep) e nível de significância (p). Os resultados obtidos foram: $\mathrm{k}=0,67$, ep $=0,38$ e $\mathrm{p}=0,041$.

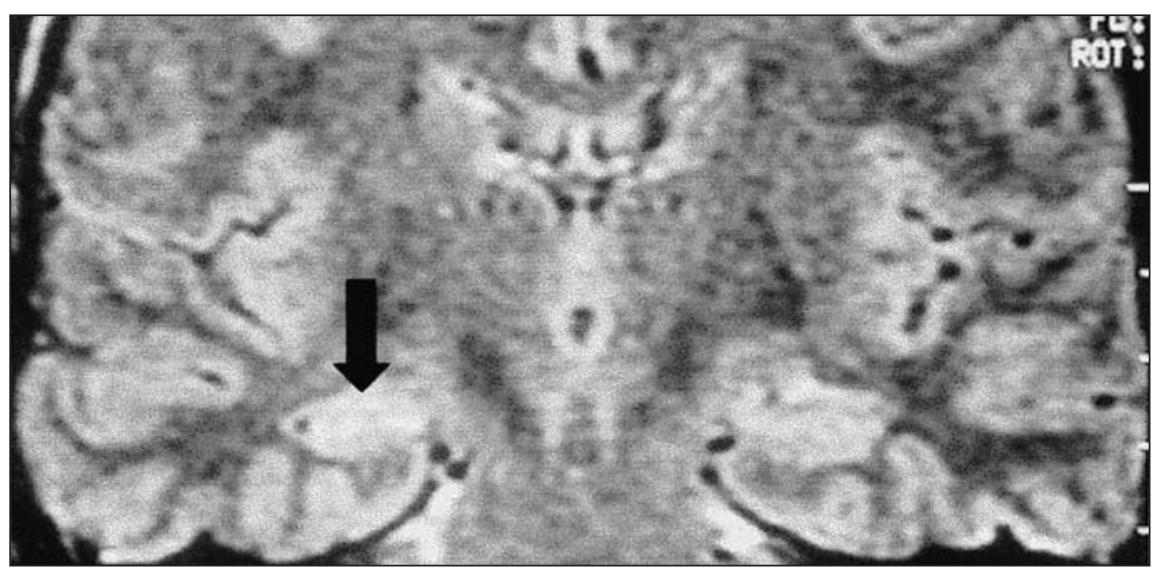

A
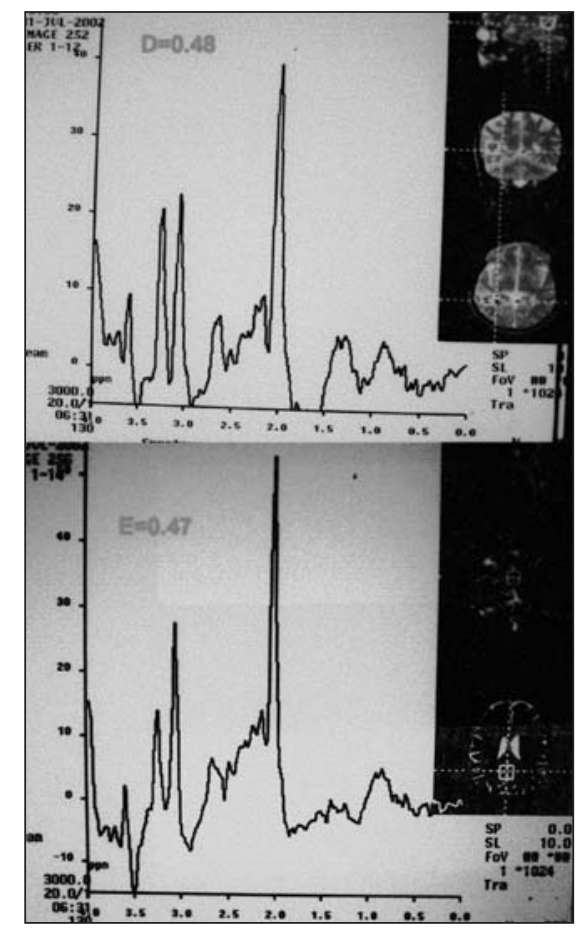

B

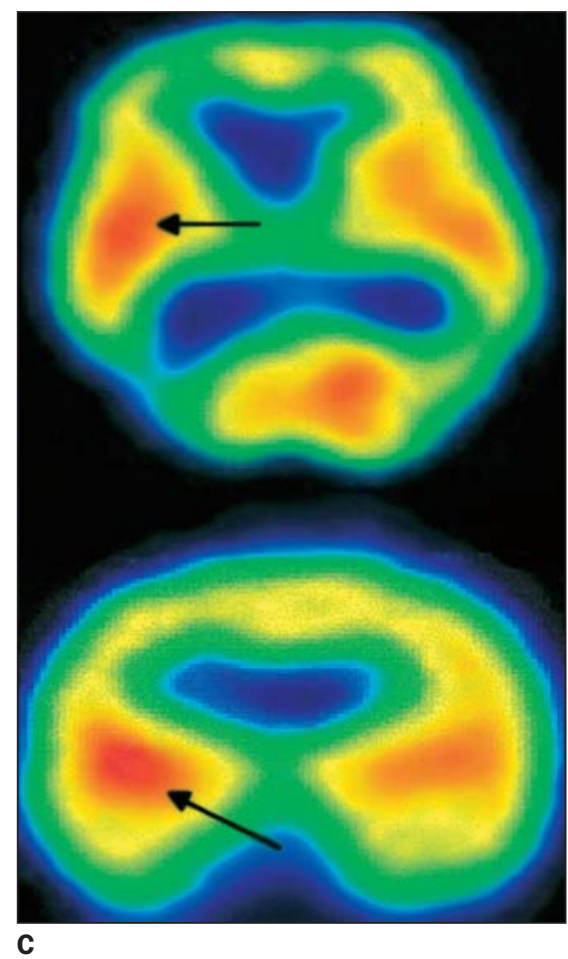

Figura 1. Caso 4: paciente do sexo masculino, 54 anos. EEG com descarga ictal temporal bilateral, predominante à direita. Em A, RM em FLAIR: esclerose mesial hipocampal direita (seta). Em B, RME: redução bilateral da relação NAA/Cho $+\mathrm{Cr}$, com discreto predomínio no hipocampo esquerdo. Em C, SPECT ictal, cortes transversal (acima) e coronal (abaixo): hiperfixação do radiofármaco no lobo temporal direito (setas). 


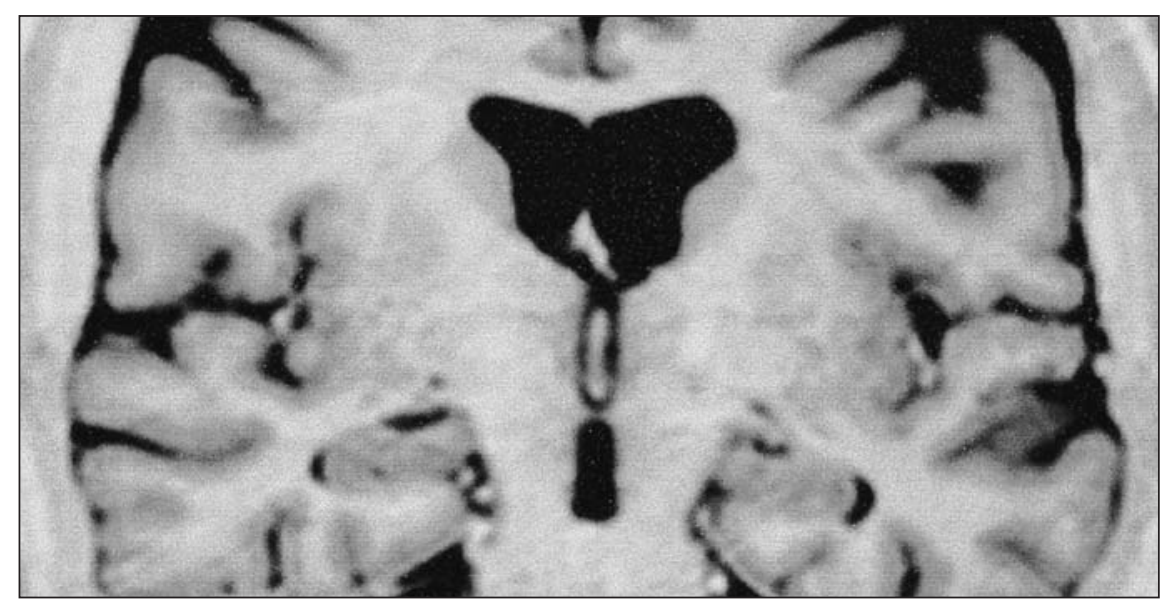

A

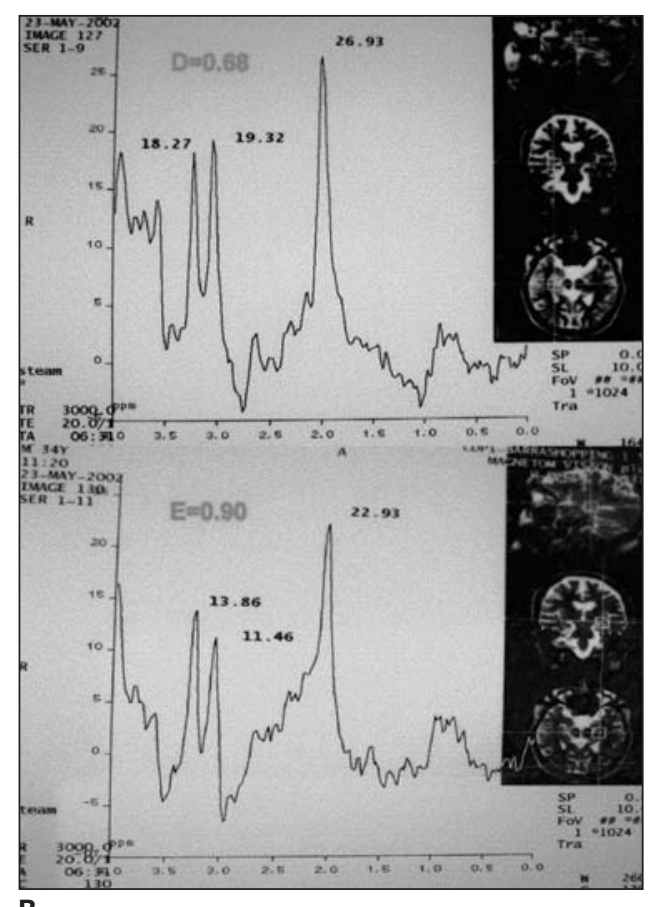

B

Figura 2. Caso 5: paciente do sexo masculino, 36 anos. EEG com descarga ictal temporal bilateral. Em A, RM em T1: ausência de alterações anatômicas. Em B, RME: relação NAA/Cho+Cr reduzida no hipocampo direito e normal à esquerda. Em C, SPECT ictal, cortes transversal (acima) e coronal (abaixo): hiperfixação do radiofármaco na porção ântero-medial do lobo temporal esquerdo (setas).

\section{DISCUSSÃO}

O SPECT cerebral ictal é um procedimento considerado útil na avaliação de pacientes com ELT, e muitos investigadores já discorreram sobre suas vantagens ${ }^{(13,14)}$. A administração do radiofármaco no período ictal, isto é, até 60 segundos do início da crise, ou no pós-ictal imediato (entre 60 e 120 segundos) resulta em uma imagem que traduz a distribuição do fluxo sanguíneo cerebral naquele momento e que permanece estável por horas. Podemos ob- ter, assim, uma imagem com área de aumento da atividade (região hipermetabólica) no foco da crise em $80 \%$ a $100 \%$ dos pacientes $^{(\mathbf{1 0 , 1 5})}$. Estudos sobre SPECT ictal reportaram valores de sensibilidade entre $81 \%$ e $93 \%$, especificidade de até $92,3 \%$ e valor preditivo positivo para a localização de focos unilaterais de até $97 \%{ }^{(\mathbf{1 0}, 15,16)}$.

$\mathrm{O}$ pequeno número de pacientes selecionados em nosso estudo resultou de alguns fatores de ordem prática, os quais tentamos explicar. O Programa de Epilepsias tem como finalidade não só a pesqui- sa clínica, mas também a prestação de serviços, assim como o Setor de vídeo-EEG. Isto torna praticamente impossível a realização de estudos voltados exclusivamente para a pesquisa, que demandam um tempo longo de internação. Dessa forma, tivemos que adequar os propósitos para que, na medida do possível, não existissem perdas de ambos os lados. Assim, os pacientes foram selecionados a partir das consultas de rotina do ambulatório de epilepsia. Inicialmente estava prevista a seleção de dez pacientes para este trabalho. Selecionamos efetivamente nove pacientes, que foram internados no Setor de vídeo-EEG para proceder à monitorização eletroencefalográfica e SPECT ictais. Entretanto, três pacientes foram excluídos: dois não apresentaram crises convulsivas mesmo com a retirada total da medicação anti-epileptogênica, e um, por motivo de fobia, não realizou a RM e a RME. A validade estatística deste estudo, realizado com número pequeno de pacientes, se reporta à ausência de estudos comparativos entre RM, RME e SPECT cerebral ictal em uma pesquisa bibliográfica que abrangeu as principais publicações indexadas entre 1996 e 2002.

Neste estudo, a duração das crises variou de 109 a 280 segundos (média de 152 segundos) e o tempo de administração do radiofármaco variou de 30 a 262 segundos do início da crise (média de 96 segundos). Acreditamos que, por se tratar de um número pequeno de pacientes, estes dados sofreram um desvio estatístico por força dos tempos de duração da crise e de administração do traçador de um único paciente (caso 3: 280 segundos e 262 segundos, respectivamente), que geraram valores médios um pouco acima dos valores encontrados na literatura. A exclusão destes valores reduz as médias de duração das crises para 126 segundos e dos tempos de administração do traçador para 63 segundos, o que condiz com os dados da literatura ${ }^{(14)}$. Este fato, entretanto, não acarretou qualquer tipo de dificuldade na interpretação visual do SPECT ictal, exceto pelo achado de uma área mais extensa de hiperfixação do radiofármaco (regiões temporal e frontal direitas). A relação entre o tempo da injeção e o padrão perfusional ictal pode ser facilmente explicada. As descargas ictais podem se espalhar para mui- 
tas regiões cerebrais e estas regiões podem, por sua vez, depender dos diferentes padrões temporais e espaciais das descargas ictais $^{(\mathbf{1 4})}$. A administração precoce do radiofármaco é o fator determinante mais importante da localização radioisotópica do foco da crise ${ }^{(\mathbf{1 4})}$. Quanto mais próxima do evento ictal for a injeção do traçador, maior a sensibilidade do método. Se o radiofármaco for injetado durante a fase inicial da crise, a propagação ictal fica restrita às estruturas circunjacentes ao foco epileptogênico e a hiperperfusão ictal subseqüente pode também ficar limitada ao lobo temporal epileptogênico ${ }^{(\mathbf{1 4})}$. Se, em contrapartida, o radiofármaco for injetado durante a fase mais tardia da crise, a propagação ictal pode se alastrar e a subseqüente hiperperfusão ictal pode ser mais extensa ${ }^{(\mathbf{1 4})}$. Assim se explica a hiperperfusão frontal direita associada ao foco temporal homolateral no caso 3 .

Para a análise estatística do estudo consideramos como resultados concordantes (4/6; 67\%) aqueles em que o SPECT ictal identificou o foco epileptogênico no mesmo lado onde havia a presença de, pelo menos, um tipo de alteração nos procedimentos radiológicos, que podia ser anatômica, bioquímica ou ambas, independentes de serem uni ou bilaterais, porém, neste último caso, levando-se em conta o lado dominante das alterações. Resultados discordantes $(2 / 6 ; 33 \%)$ foram aqueles em que o SPECT ictal identificou o foco epileptogênico no lado oposto às alterações descritas à RM e/ou RME, levando-se em conta a dominância das alterações bilaterais. Assim, conseguimos uma correlação entre os dois estudos. Contudo, faz-se necessário tecer algumas considerações com base em análise mais minuciosa dos casos.

Sabemos que os métodos não-invasivos de investigação diagnóstica dos focos epileptogênicos possuem algumas limitações. Na maioria dos trabalhos de pesquisa clínica o EEG ictal foi considerado o método padrão-ouro para comparações com o SPECT ictal ou com a RM, porém o EEG de superfície possui suas limitações próprias em relação à precisão, pois a monitorização do escalpo cobre apenas uma parte do cérebro e as alterações eletroencefalográficas requerem um certo número de neurônios sincrônicos, o que muitas vezes determina a necessidade de estudos eletrofisiológicos invasivos ${ }^{(\mathbf{1 4 , 1 6})}$. Mesmo esses estudos invasivos podem não ser úteis ou levar a diagnósticos incorretos, uma vez que existe a dependência da escolha correta das regiões corticais a serem monitorizadas pelos eletrodos. Esta limitação é amplamente reconhecida, particularmente quando a RM convencional não mostra alterações estruturais $^{(\mathbf{1 6})}$. Neste sentido, o SPECT ictal pode ser considerado uma técnica de avaliação pré-cirúrgica independente e confirmatória ${ }^{(14)}$. O consenso internacional considera que a concordância de resultados entre dois métodos de investigação é suficiente para o diagnóstico do foco epileptogênico ${ }^{(\mathbf{1 6})}$.

Neste trabalho consideramos que o EEG ictal foi diagnóstico nos casos de ELT unilateral (casos 1, 2 e 5), parcialmente diagnóstico no único caso de ELT bilateral em que se determinou a dominância das descargas temporais (caso 4), e não diagnóstico nos demais casos de ELT bilateral sem dominância (casos 3 e 6).

Nos casos de EEG ictal diagnóstico a RM identificou alteração anatômica, isto é, esclerose mesial hipocampal, unilateral, em lado concordante (à direita), em dois casos, sendo que em um (caso 1) a RME foi normal e o SPECT ictal concordante com o EEG ictal e com a RM (lobo temporal direito); em outro (caso 6), a RME mostrou alteração bioquímica bilateral com predomínio à direita e o SPECT ictal identificou o foco no lobo temporal esquerdo. No caso 2 a RM mostrou alteração anatômica bilateral, assim como a RME, havendo, nesta última, predomínio da alteração bioquímica no lado esquerdo. O SPECT ictal identificou o foco em lobo temporal esquerdo, concordante com o EEG ictal e com a RME.

Nos casos de EEG ictal parcialmente diagnóstico ou não diagnóstico a RM foi normal em dois casos, sendo que em um (caso 5) a RME foi marcadamente alterada em hipocampo direito (esquerdo normal) e o SPECT ictal identificou o foco em lobo temporal esquerdo; em outro (caso 3), a RME mostrou alteração bioquímica bilateral, com predomínio à direita, e o SPECT ictal identificou o foco em lobo temporal direito. No terceiro caso (caso 4), em que o EEG ictal mostrou dominância da descarga neuronal à direita, a RM evidenciou esclerose mesial hipocampal direita, a RME foi alterada bilateralmente, com uma diferença de resultados desprezível entre ambos os hipocampos (0,47 à esquerda e 0,48 à direita) e o SPECT ictal identificou o foco em lobo temporal direito.

Estes achados nos levam a considerar que, para a determinação do foco epileptogênico, os métodos de investigação diagnóstica (EEG, RM, RME e SPECT ictal) não devem ser analisados de forma independente entre si, isto é, a importância dos métodos reside na interpretação conjunta de todos os resultados, uma vez que todos apresentam limitações inerentes ao próprio método ou à fisiopatologia de propagação da descarga neuronal. Além das limitações do EEG inerentes ao método, este se torna não diagnóstico nos casos de ELT bilateral, ressaltando-se a necessidade de avaliação minuciosa dos traçados eletroencefalográficos no sentido de se determinar a dominância das descargas neuronais, nos casos em que isto é possível, para que a interpretação dos resultados dos demais exames complementares seja significati$\mathrm{va}^{(\mathbf{1 4})}$. Mesmo a RME não parece ser um método diagnóstico que possa ser utilizado de forma independente na determinação do foco epileptogênico, por causa da variação de achados anatômicos que pode ocorrer nos casos de ELT, isto é, podemos ter avaliações anatômicas normais e achados de esclerose mesial hipocampal uni ou bilateral. A associação da espectroscopia de prótons à RM aumentou a sensibilidade do método na avaliação de muitas doenças neurológicas e psiquiátricas. Em nosso estudo, entretanto, observamos alguma divergência entre os resultados anatômicos e bioquímicos. Obtivemos resultados normais na RM com RME alterada uni e bilateralmente (casos 3 e 5), assim como RM alterada com RME normal (caso 1). A sensibilidade da RM na ELT bilateral aumentou com o achado de comprometimento bioquímico unilateral, apesar do EEG não ter sido diagnóstico, em que o hipocampo contralateral foi considerado dentro da faixa de normalidade (caso 5). O comprometimento bioquímico em ambos os hemisférios não influiu de forma importante na determinação do foco em outro caso de ELT bilateral, sem dominância 
eletroencefalográfica, uma vez que os resultados não apresentaram diferença significativa entre si $(0,02)$ (caso 3 ).

Nos casos de RM alterada unilateralmente, em todos os casos mostrando esclerose mesial hipocampal direita (casos 1,4 e 6), a RME apresentou resultados normais (caso 1) ou foi concordante, demonstrando comprometimento em ambos os hemisférios (casos 4 e 6), sendo um com dominância (caso 6, diferença de 0,08 entre os lados) e outro sem dominância (caso 4). Observamos, então, que nesses casos não houve, necessariamente, um acréscimo positivo de informações em relação ao EEG ictal e à RM.

No caso de RM mostrando alteração bilateral a RME também foi concordante, demonstrando comprometimento em ambos os hemisférios, com predomínio de lateralização à esquerda (caso 2, diferença de 0,05 entre os lados). Se considerarmos esta diferença significativa, podemos dizer que houve aumento da sensibilidade do método radiológico, pois neste caso a dominância da alteração bioquímica se relacionou positivamente com o traçado eletroencefalográfico.

No contexto do nosso estudo o SPECT ictal pode ser considerado um procedimento importante na avaliação das ELT refratárias. Entretanto, seu uso como método de investigação diagnóstica deve ser racional, dentro de uma hierarquia de achados clínicos, eletroencefalográficos, anatômicos e bioquímicos. Isto se deve ao fato não só do SPECT ictal, como diz o nome, ser realizado a partir da injeção do radiofármaco em pacientes durante uma crise convulsiva, o que nem sempre é agradável para esses pacientes, como também pelo fato de que a sua realização envolve problemas logísticos. Por problemas logísticos entende-se a organização de infra-estrutura específica para o exame, isto é, o SPECT ictal deve ser realizado em um ambiente hospitalar, envolvendo colaboração multidisciplinar (neurologistas, médicos nucleares, radiofarmacêuticos e equipe de enfermagem) e dentro de um esquema rígido de preparação, transporte e utilização de materiais radioativos.

A análise dos resultados apresentados em nosso trabalho mostrou que a importância do SPECT ictal reside nos casos em que há discordância ou indefinição na análise comparativa dos resultados obtidos pelo EEG ictal, RM e RME. Dentro deste contexto, podemos dizer que o SPECT ictal se faz necessário marcadamente nos casos de ELT bilateral, com ou sem dominância, em que os resultados combinados dos procedimentos radioisotópico e radiológicos (RM e RME) definem a lateralização do foco epileptogênico, e nos casos de ELT unilateral, em que as alterações anatômicas e bioquímicas são bilaterais.

Neste estudo obtivemos dois resultados de SPECT ictal discordantes $(2 / 6 ; 33 \%)$. Esta porcentagem pode, a princípio, parecer elevada, considerando-se o pequeno número de pacientes estudados. Entretanto, no levantamento bibliográfico de estudos comparativos entre os procedimentos radiológico e radioisotópico, observamos que o número de pacientes submetidos ao SPECT ictal é, em geral, menor do que os que são submetidos à RM e que, apesar do procedimento radiológico não incluir a RME, o que poderia aumentar a sensibilidade do estudo, o total de casos discordantes não difere significativamente, em números porcentuais, dos achados deste trabalho. Como exemplo, podemos citar o estudo de Oliveira et al. ${ }^{(\mathbf{1 6})}$, com casuística de 48 pacientes com ELT, todos submetidos à RM, dos quais apenas 14 (29\%) foram submetidos ao SPECT ictal, encontrando-se resultados concordantes em dez pacientes $(71 \%)$ e discordantes em quatro (29\%). Acreditamos que o porcentual de casos discordantes um pouco mais elevado neste nosso trabalho está relacionado ao pequeno número de casos e não ao procedimento radioisotópico em si.

No primeiro caso (caso 5) o EEG ictal não foi diagnóstico (ELT bilateral), a RM foi normal e a RME mostrou alteração marcadamente à direita, ao passo que o SPECT ictal detectou o foco à esquerda. $\mathrm{Na}$ verdade, exceto pelo fato da ausência de alterações anatômicas e bioquímicas à esquerda, pode-se considerar o resultado do procedimento radioisotópico como aceitável em relação ao traçado do EEG ictal, que demonstrou descaras temporais bilaterais no início da crise convulsiva. Neste caso, poderíamos dizer que o SPECT ictal detectou o lado dominante das descargas ictais, indeterminado no traçado ele- troencefalográfico. Uma outra explicação para este fato, bem descrita na literatura ${ }^{(\mathbf{1 6})}$, é a possibilidade de uma propagação extremamente rápida da descarga ictal para o lobo contralateral. Esta explicação não se aplica ao nosso trabalho por duas razões: em primeiro lugar, isto ocorre quando a administração do traçador se faz a partir de 90 segundos do início da crise e, neste caso, a injeção foi aos 62 segundos da crise; em segundo lugar, a imagem radioisotópica obtida é uma área extensa de hiperfixação do radiofármaco, que traduz a área de propagação da descarga ictal e, neste caso, a imagem obtida foi focal, bem localizada na porção ântero-medial do lobo temporal esquerdo. Uma boa medida, nestes casos, é a realização de estudos radioisotópicos ictais seriados, para melhor avaliação diagnóstica.

No segundo caso discordante (caso 6) o EEG ictal determinou a descarga em lobo temporal direito, a RM evidenciou esclerose mesial hipocampal direita e a RME foi alterada bilateralmente, com predomínio à direita. O SPECT ictal detectou área de hiperfixação do traçador em lobo temporal esquerdo. Segundo o consenso internacional, a realização do SPECT ictal, neste caso, seria dispensável, já que os dois métodos de investigação prévios foram concordantes. Porém, em face dessa discordância, este paciente foi novamente submetido ao SPECT ictal que, mais uma vez, mostrou área de hiperfixação em lobo temporal esquerdo. A injeção do traçador, neste caso, foi feita aos 30 segundos da crise, o que afasta a possibilidade de propagação rápida da descarga ictal. Uma explicação plausível pode ser a de que pacientes com ELT ocasionalmente podem apresentar descargas temporais independentes $^{(\mathbf{1 6})}$. Assim, não se pode excluir a possibilidade de que este episódio ictal em particular pode ter se originado no lobo temporal contralateral.

Em relação ao radiofármaco utilizado neste trabalho, o ${ }^{99 \mathrm{~m}} \mathrm{Tc}-\mathrm{ECD}$, sendo um traçador lipossolúvel e neutro, de fácil preparo, de alta pureza radioquímica, estabilidade e retenção intracerebral prolongadas, com excelente relação de fixação entre substância cinzenta e branca $(1,5: 1$, respectivamente), tem provado ser um agente de perfusão cerebral superior, mar- 
cadamente nos estudos ictais de pacientes com ELT refratária, que relatam resultados altamente comparáveis aos obtidos com HMPAO (hexametilpropilenoaminoxima) em termos de precisão na localização do foco epileptogênico ${ }^{(\mathbf{1 6}, \mathbf{1 7})}$.

Este estudo foi limitado pelo pequeno número de pacientes, devendo ser enfocado como uma análise preliminar da comparação entre resultados de SPECT ictal, RM e RME nos casos de ELT refratária. Assim, deve-se realizar um número maior de estudos comparativos para que se possa obter uma análise estatística mais abrangente sobre o assunto em questão.

\section{REFERÊNCIAS}

1. Guerreiro CA, Guerreiro M, Cendes F, et al. Epilepsia. São Paulo: Lemos Editorial, 2000:1-10.

2. Garnett WR. Epilepsy. In: Dipiro JT, Talbert RL,
Hayes PC, et al., eds. Pharmacotherapy: a pathophysiologic approach. New York, NY: Elsevier, 1989:611-31.

3. Internet: www.aspe.hc.unicamp.br/epilepsia.shtml

4. Shorvon SD. Epidemiology, classification, natural history, and genetics of epilepsy. Lancet 1990;336: 93-6.

5. Internet: neurologia.rediris.es/congreso-1/conferencias/epilepsia-1.html

6. Kuhl DE, Edwards RQ. Image separation radioisotope scanning. Radiology 1963;80:653-62.

7. Assessment of brain SPECT. Report of the Therapeutics and Technology Assessment Subcommittee of the American Academy of Neurology. Neurology 1996;46:278-85.

8. Cascino GD. Advances in neuroimaging: surgical localization. Epilepsia 2001;42:3-12.

9. Connelly A, Jackson GD, Duncan JS, King MD, Gadian DG. Magnetic resonance spectroscopy in temporal lobe epilepsy. Neurology 1994;44:1411-7.

10. Catafau AM. Brain SPECT clinical practice. Part I: perfusion. J Nucl Med 2001;42:259-71.

11. Chang DJ, Zubal IG, Gottschalk C, et al. Comparison of statistical parametric mapping and SPECT difference imaging in patients with temporal lobe epilepsy. Epilepsia 2002;43:68-74.

12. Diegues MEM, Pellini MP, Leon SVA, et al. Correlação entre SPECT ictal e RME interictal para a localização do foco epileptogênico em pacientes com ELT de difícil controle. PO-131. Arq NeuroPsiquiatr 2002;80:88-9.

13. Ho SS, Berkovic SF, Berlangieri SU, et al. Comparison of ictal SPECT and interictal PET in the presurgical evaluation of temporal lobe epilepsy. Ann Neurol 1995;37:738-45.

14. Lee SK, Lee SH, Kim SK, Lee DS, Kim H. The clinical usefulness of ictal SPECT in temporal lobe epilepsy: the lateralization of seizure focus and correlation with EEG. Epilepsia 2000;41:955-62.

15. Camargo EE. Brain SPECT in neurology and psychiatry. J Nucl Med 2001;42:611-23.

16. Oliveira AJ, Costa JC, Hilario LN, Anselmi OE, Palmini A. Localization of the epileptogenic zone by ictal and interictal SPECT with ${ }^{99 \mathrm{~m} T c-e t h y l ~ c y s t e-~}$ inate dimer in patients with medically refractory epilepsy. Epilepsia 1999;40:693-702.

17. Lee DS, Lee SK, Kim YK, et al. Superiority of HMPAO ictal SPECT to ECD ictal SPECT in localizing the epileptogenic zone. Epilepsia 2002;43: 263-9. 